\title{
Synchrotron emission from circumstellar disks around massive stars
}

\author{
Y. A. Shchekinov ${ }^{1,2}$ and A. M. Sobolev ${ }^{3}$ \\ 1 Department of Physics, Rostov State University, Rostov on Don 344090, Russia \\ 2 Isaac Newton Institute of Chile, Rostov on Don Branch, Russia \\ 3 Astronomical Observatory, Ural State University, Ekaterinburg 620083, Russia \\ e-mail: Andrej.Sobolev@usu.ru
}

Received 20 December 2003 / Accepted 13 February 2004

\begin{abstract}
We argue that the interaction of stellar wind with the surface of a circumstellar accretion (or protoplanetary) disk can result in the acceleration of relativistic electrons in an external layer of the disk, and produce synchrotron radiation. Conservative estimates give a total synchrotron luminosity $L_{\mathrm{s}} \sim 10^{-5} L_{\odot}$ for a central star with $\dot{M}=10^{-6} M_{\odot} \mathrm{yr}^{-1}$, comparable with the value observed around the TW object in the $\mathrm{W} 3(\mathrm{OH})$ region.
\end{abstract}

Key words. accretion, accretion disks - stars: circumstellar matter - acceleration of particles

\section{Introduction}

Recent investigations have shown that $\mathrm{H}_{2} \mathrm{O}$ masers in the vicinity of young stellar objects can be associated both with jets and circumstellar disks (see Torrelles et al. 2002 for the most recent review). It is suggested that systems in which $\mathrm{H}_{2} \mathrm{O}$ masers trace disks are less evolved than those in which masers trace outflows.

The detection of synchrotron emission at $v=1.6-14.7 \mathrm{GHz}$ from a very young massive stellar object associated with $\mathrm{H}_{2} \mathrm{O}$ masers in the $\mathrm{W} 3(\mathrm{OH})$ region (Turner \& Welch 1984, hereafter TW object) demonstrated the presence of relativistic electrons in the region nearly coincident with the location of the $\mathrm{H}_{2} \mathrm{O}$ masers (Reid et al. 1995). These authors interpreted the synchrotron emission as related to acceleration of the relativistic electrons in shock waves associated with a powerful jet with E-W orientation emanating from the young stellar object (Reid et al. 1995; Wilner et al. 1999). Association with the jet is based on the measurements of proper motions of the $\mathrm{H}_{2} \mathrm{O}$ masers which are compatible with the hypothesis of a bipolar outflow moving in the E-W direction (Alcolea et al. 1993). We argue that interpretation of proper motions pattern of the $\mathrm{H}_{2} \mathrm{O}$ maser by Alcolea et al. (1993) is not unique (a similar situation is described in Fiebig et al. 1996) and does not rule out the hypothesis of formation of the $\mathrm{H}_{2} \mathrm{O}$ masers on the surface of the circumstellar disk or in a molecular ring seen edge-on. Evidence pro and contra the disk hypothesis are presented throughout paper.

Interstellar $\mathrm{H}_{2} \mathrm{O}$ masers are usually located in regions influenced by strong MHD shocks emanating from young stellar

Send offprint requests to: Y. A. Shchekinov,

e-mail: yus@phys.rsu.ru objects. These shocks affect the boundaries of the jets, outflows, the surface of circumstellar disks and the parts of the ambient molecular core that are closest to the young stellar object. So one might, in principle, expect that the acceleration of relativistic electrons generating synchrotron emission can arise not only at the jet boundaries and can be a common phenomenon for the vicinities of $\mathrm{H}_{2} \mathrm{O}$ masers. The question therefore arises whether synchrotron emission at locations close to $\mathrm{H}_{2} \mathrm{O}$ masers does necessarily originate in jets or if an alternative explanation connected with high energy processes in circumstellar disks is possible.

In this paper we propose estimates which show that the interaction of stellar wind from a central star with an accretion disk can support favorable conditions for the acceleration of electrons and the generation of synchrotron radiation from relatively narrow boundary layers on the disk surface. We show that this interaction can produce a total synchrotron luminosity in the TW object comparable to what is observed. In Sect. 2 we present simple estimates of the acceleration mechanism, and calculate the resulting synchrotron emission from the disk surface, in Sect. 3 we describe the spatial distribution of the synchrotron radiation, in Sect. 4 we provide arguments in favor of a circumstellar disk around the TW object, Sect. 5 summarizes the results.

\section{Energy requirements}

Strong winds from early-type stars generate through hydrodynamical instabilities numerous shocks with Mach numbers up to 20 (Carlberg 1980; Owocki \& Rybicki 1984). The density of shocks increases near the disk surface due to the Kelvin-Helmholtz $(\mathrm{KH})$ instability of the shear flow between 
the wind and the surface, and subsequent interaction of the wind with the vortices and coherent structures generated by the instability. On nonlinear stages these structures have sizes and spacing of the order of the thickness of the mixing layer (Roshko 1976; Head \& Bandyopadhyay 1981): $\delta \sim \sqrt{4 \eta r / v_{\infty}}$. Assume that the energy density of the shocks in a layer of thickness $\Delta H(r)$ is a fraction $\beta$ of the wind energy density: $\varepsilon_{\mathrm{s}}=\beta \rho v_{\infty}^{2} / 2$, where

$\rho=\frac{\dot{M}}{4 \pi r^{2} v_{\infty}}$

$v_{\infty}$ is the terminal velocity of the wind, $\dot{M}$, the mass loss rate. The total energy of shocks in the boundary layers from both sides of the disk is

$E_{\mathrm{s}}=\frac{\beta \dot{M} v_{\infty}}{2} \int_{R_{*}}^{R} \frac{\Delta H(r)}{r} \mathrm{~d} r$

where $R_{*}$ is the stellar radius, $R$, the disk radius. Assume that $\Delta H(r)=h r, h \ll 1$. Then

$E_{\mathrm{s}}=10^{44} \beta h \dot{M}_{-6} v_{8} R_{3} \mathrm{erg}$,

where $\dot{M}_{-6}=\dot{M} /\left(10^{-6} M_{\odot} \mathrm{yr}^{-1}\right), v_{8}=v_{\infty} /\left(10^{8} \mathrm{~cm} \mathrm{~s}^{-1}\right), R_{3}=$ $R /\left(10^{3} \mathrm{AU}\right)$ (note that the radius of the synchrotron structure observed by Reid et al. 1995 is of the order of $\left.10^{3} \mathrm{AU}\right)$.

Charged particles will be accelerated by the shocks through the first order Fermi mechanism. Assume that the mechanical energy of shock waves is transformed into cosmic ray energy until the nonlinear back reaction of accelerated particles leads to significant dissipation of the large-scale turbulence (Zank et al. 1990). Under these conditions one can expect that in the steady state the energy accumulated in non-thermal ions will be comparable to the energy stored in shocks, while the fraction of energy contained in relativistic electrons is $\left(m_{\mathrm{e}} / m_{\mathrm{p}}\right)^{(q-1) / 2}$, $q$ being the exponent of a power law spectrum of relativistic electrons (see below). The maximum energy of non-thermal ions $e_{\mathrm{m}}$ is found from the condition (Bykov \& Fleishman 1992)

$$
\frac{3 u l_{\mathrm{s}}}{v \Lambda} \sim 1
$$

where $u$ is the shock speed, $l_{\mathrm{s}}$ is the distance between the shocks, $v$ is the particle velocity, $\Lambda$ is the transport length (Toptygin 1985; Bykov \& Fleishman 1992)

$\Lambda \simeq 0.3 l_{\mathrm{s}}\left(r_{b} / l_{\mathrm{s}}\right)^{2-\xi}$

where $r_{b}=p c / e B, \xi$ is the power-law exponent of the fluctuating magnetic field spectrum $\mathrm{d}\left\langle b^{2}\right\rangle / \mathrm{d} k \sim k^{-\xi}$ at $k l_{\mathrm{s}} \gg 1$ (Bykov $\&$ Fleishman 1992). The mean distance between the shocks in strong radiation-driven winds is of the order $l_{\mathrm{s}} \sim 10^{-2} R_{*}\left(r / R_{*}\right)^{2}$ (Lucy 1982; White 1985), and can reach $l_{\mathrm{s}} \sim 0.1-10 \mathrm{AU}$ at $r \sim 1-10 \mathrm{AU}$ for an $\mathrm{O}-$ or early B-type star. The actual value can be smaller if multiple reflections of the shocks on the vortices and coherent structures in the boundary layer of the disk are taken into account. As a conservative estimate one can take $l_{\mathrm{S}} \sim 1 \mathrm{AU}$. This results in $e_{\mathrm{m}} \sim 300 \mathrm{GeV}$ for $\xi=1.5$, and $e_{\mathrm{m}} \sim 10 \mathrm{GeV}$ for $\xi=1.7$. We will assume that the energy spectrum of non-thermal electrons extends also to these values,
So that the characteristic frequency $v_{b}=2 \pi \gamma e B / m_{\mathrm{e}} c^{2}$, where $\gamma=e / m_{\mathrm{e}} c^{2}$, can be as high as $20 \mathrm{GHz}$.

With these assumptions and for a power-law spectrum of relativistic electrons

$N(e) \mathrm{d} e=\kappa \mathrm{e}^{-q} \mathrm{~d} e, e \geq e_{\min }, q>2$,

where $\kappa=(q-2) \varepsilon_{\mathrm{s}}\left(m_{\mathrm{e}} / m_{\mathrm{p}}\right)^{q-2 / 2} e_{\min }^{q-2}$, with $q=2.2$ as observed in the TW object and $e_{\min }=\gamma_{\min } m_{\mathrm{e}} c^{2}$, one can obtain the total synchrotron luminosity

$L_{\mathrm{sD}}=\int^{14.7 \mathrm{GHz}} L_{\mathrm{s}}(v) \mathrm{d} v=10^{-3} \beta h \gamma_{\min }^{0.2} \dot{M}_{-6} v_{8} R_{3} B_{0.01}^{1.6} L_{\odot}$,

where $B_{0.01}=B /(0.01 \mathrm{G})$. The synchrotron luminosity of the TW object in the frequency range $v=1.6-14.7 \mathrm{GHz}$ is $L \cong 5 \times$ $10^{-6} L_{\odot}$, as inferred from Reid et al. (1995). It is seen that at fiducial values of the parameters $\beta h \sim 5 \times 10^{-3} \gamma_{\text {min }}^{-0.2}$ provides a synchrotron luminosity from the boundary layer comparable to the luminosity observed in the TW object. Note, that with the same assumptions about the generation of relativistic electrons in the jet with a collimation angle $h$ its total synchrotron luminosity is $L_{\mathrm{sJ}}=h L_{\mathrm{sD}} \ll 1$, so that the observed luminosity of the TW object can be understood only if $\beta h^{2} \sim 5 \times$ $10^{-3} \gamma_{\min }^{-0.2}$, i.e. under the jet hypothesis a much larger (by a factor $1 / h \gg 1$ ) fraction of the wind energy should be transformed into the shocks which accelerate electrons.

\section{Angular size versus frequency}

Reid et al. (1995) found that the angular size of the synchrotron source decreases with frequency, and attributed it to self-absorption of synchrotron photons. In our model the maximal (along radius) optical depth is

$\tau=2 \int_{r_{0}}^{R} \mu(r) \mathrm{d} r$

where $r_{0}$ is the inner disk radius, $\mu$ is the absorption coefficient for synchrotron emission (see Ginzburg 1975)

$\mu=g(q) \frac{e^{3}}{2 \pi m_{\mathrm{e}}}\left(\frac{3 e}{2 \pi m_{\mathrm{e}}^{3} c^{5}}\right)^{q / 2} \kappa B^{(q+2) / 2} v^{-(q+4) / 2}$,

$g(q) \simeq 0.7$ for $q \simeq 2$ (see Ginzburg 1975). This gives for the above assumption about the energy density in relativistic electrons $\varepsilon(r)=\beta \dot{M} v_{\infty} / 8 \pi r^{2}$, and for $B=B_{0}\left(r_{0} / r\right)^{b}$ optical depth

$\tau=3 \times 10^{8} \beta B_{0,0.01}^{(q+2) / 2} \dot{M}_{-6} r_{0}^{-1}$, at $v=10 \mathrm{GHz}$,

and

$\tau=4 \times 10^{11} \beta B_{0,0.01}^{(q+2) / 2} \dot{M}_{-6} r_{0}^{-1}$, at $v=1 \mathrm{GHz}$,

and obviously $\tau$ is much less than one for $r_{0}>R_{*}$. One can show that free-free absorption is also negligible for the parameters of interest. Note that for the best fit parameters derived by Reid et al. within the jet model $\left(e_{\min }=15 m_{\mathrm{e}} c^{2}\right.$, the total density of relativistic electrons $\int N(e) \mathrm{d} e=5 \times 10^{-3} \mathrm{~cm}^{-3}$, $\left.B_{0}=0.01 \mathrm{G}\right)$ Eq. (9) gives optical depth $\tau \sim 10^{-22} L \ll 1$ 
at $v=1 \mathrm{GHz}, L$ being the characteristic size of the absorbing region. Under such conditions the decrease of the angular size of the source with frequency (approximately as $\theta \propto v^{-1}$, Reid et al. 1995) can be explained only by assuming that the spectral properties of the synchrotron emission vary with radius. For a power-law spectrum of relativistic electrons as given by Eq. (6) in the energy interval $e=\left[e_{\min }, e_{\max }\right]$ the synchrotron emissivity is approximately a power-law $I_{v} \propto v^{-(q-1) / 2}$ in the frequency interval $v=0.29\left[v_{\min }, v_{\max }\right]$, where $v_{\min \text { max }}$ correspond to minimal and maximal energies of electrons. Below and above this interval the emissivity varies as $I_{v} \propto\left(v / v_{\min }\right)^{(3 q-1) / 6}$, and $I_{v} \propto\left(v / v_{\max }\right)^{(q-2) / 2} \exp \left(-v / v_{\max }\right)$, respectively. The mean frequency in the spectrum is determined by $q, v_{\min }$ and $v_{\max }$, and $\bar{v}=(3-q) v_{\max } /(5-q)$ for $q<3$ and $v_{\max } \gg v_{\min }$. The observed size of a synchrotron source will then decrease with frequency if the mean frequency in the spectrum $\bar{v}$ decreases with radius: at fixed frequency $v$ the main contribution to the observed flux comes from a region with radius corresponding to the condition $v<0.29 v_{\max }(r)$.

For fixed boundary energies $e_{\min }$ and $e_{\max }$ in the spectrum (6) the dependence of the critical frequencies $v_{\text {min }}$ and $v_{\max }$ on radius is determined by the magnetic field $B(r)$. The latter can be taken from observations of $B$ in $\mathrm{H}_{2} \mathrm{O}$ maser sources by Fiebig \& Güsten (1989) (see also Liljestroem \& Gwinn 2000 for more recent measurements)

$B \sim 60\left(\frac{n T}{10^{12} \mathrm{~cm}^{-3} \mathrm{~K}}\right)^{1 / 2} \mathrm{mG}$.

For a standard $\alpha$-disk (see Pringle 1981) with temperature

$T \simeq\left(\frac{3 G M \dot{M}_{d}}{8 \pi \sigma r^{3}}\right)^{1 / 4}, \quad r \gg R_{*}$,

and kinematic viscosity

$\eta \simeq \alpha c_{\mathrm{s}} H \simeq \alpha c_{\mathrm{s}}^{2} r \sqrt{\frac{r}{G M}}, \quad \alpha \leq 1$,

one obtains

$\rho=\frac{\Sigma}{H} \simeq \frac{\dot{M}_{d}}{3 \pi \eta H}$

here $M$ is the mass of a central star, $\dot{M}_{d}$, the accretion rate, $c_{\mathrm{s}}$ is the sound speed in the disk, $R_{*}$ is the star radius, the other notations have their usual meaning. When $T$ from Eq. (13) and $\rho$ from Eq. (15) are substituted in Eq. (12), one obtains $b=21 / 16$

$B \propto r^{-21 / 16}$.

Following the above arguments we arrive at $r \propto v^{-16 / 21}$ for $e_{\max }=$ const. This is slightly weaker than observed in the TW object, however, given the observational uncertainties and the rough character of these estimates, the obtained correspondence between theoretical and observed size-frequency dependence can be considered as satisfactory. One should mention in this connection that a possible deviation of $\Sigma(r)$ from that predicted for standard steady $\alpha$-disks (see, Nelson et al. 2000), and radial variations of the electron spectrum may contaminate this correspondence. It is worth noting in this regard, that when relativistic electrons are produced by a jet impinging on a dense ambient gas, an increase in surface brightness of the synchrotron emission is expected in the regions where the jet produces bow shocks and electrons are efficiently accelerated (in our case, these are the east and west ends of the synchrotron structure). When being accelerated in the bow shocks relativistic electrons can diffuse and fill the jet itself; however, their energy decreases and thus the surface brightness drops at lower radius (see for discussion Begelman et al. 1984). In such conditions, an inverse dependence of the angular size on frequency, i.e. the angular size increasing with frequency, should hold.

\section{Synchrotron structure around TW object: Disk versus jet}

\subsection{Dust emission: Disk}

Wyrowski et al. (1999) found that the synchrotron source in the vicinity of the TW object is associated with a thermally radiating dust cloud which also has a E-W elongated morphology. The total luminosity of the cloud in the whole range of wavelengths $\sim 4 \times 10^{4} L_{\odot}$ is estimated by them from an upper limit on the contribution of the dust emission of $0.5 \mathrm{Jy}$ at $220 \mathrm{GHz}$ and the distribution of the rotation temperature of HNCO. It is obvious that such a luminosity cannot stem from interaction of the stellar wind, whose mechanical luminosity is $\sim 150 \dot{M}_{-6} v_{8}^{2} L_{\odot}$, with the disk. It is worth noting, however, that the observed thermal dust emission in the $220 \mathrm{GHz}$ range $\left(\sim 0.3 L_{\odot}\right.$ at the assumed distance to the TW object of $2.2 \mathrm{kpc}$ ) is only a small fraction $\left(\sim 2 \times 10^{-3}\right)$ of the mechanical luminosity.

The most striking finding they mentioned is that one of the three brightest spots of dust emission is coincident with the center of the synchrotron source. Wyrowski et al. (1999) argue that this coincidence confirms that the hot dust originates from interaction of the "synchrotron jet" suggested by Reid et al. (1995) with the dense core gas confining the jet. In the disk model the observed similarity in morphologies of the synchrotron and dust emission, and particularly, the coincidence of their geometric centers, may indicate that both are connected with geometry of the dominant disk-like flow. The E-W alignment of the dust emission and the synchrotron source, the three brightest dust spots (A, B and C in Wyrowski et al. 1999 nomenclature), and molecular emission (such as $\mathrm{CH}_{3} \mathrm{CH}_{2} \mathrm{CN}, \mathrm{CH}_{3} \mathrm{OH}, \mathrm{HCOOCH}_{3}, \mathrm{H}_{2} \mathrm{CO}$, and $\mathrm{SO}_{2}$, see Fig. 3 in Wyrowski et al. 1999) can be understood within a picture of a circumstellar disk embedded in a parent cloud and rotating with angular velocity perpendicular to this direction.

\subsection{Dust emission: Jet}

Reid (private communication) mentions, however, another possible explanation: the dust giving rise to spot A (the brightest of the three) is heated by radiation of the massive star producing the synchrotron jet, while the other two (B and $\mathrm{C}$, which are located approximately on the continuation of the synchrotron emission to the west) are connected with the other massive stars. Note, however, that the hypothesis of the A, B and C spots arising due to energy radiated by embedded stars does not contradict the disk model itself. 


\subsection{Kinematics of water masers: Disk}

Fiebig et al. (1996) have shown that the maser kinematics admits an ambiguous interpretation: it can be explained both within the disk and the outflow hypothesis. We argue that this ambiguity exists in the case of the TW object as well.

Alcolea et al. (1993) showed that the distribution of the maser spots in $\mathrm{W} 3(\mathrm{OH})$ consists of 3 major clusters - one in the center plus eastern and western clusters located at approximately the same distance from the center. This is reminiscent of the masers which appear in the disk with the bright source in the center and a central decrease in the distribution of masing water. Such a situation in the close vicinity of the TW object is quite plausible. However, careful analysis of the situation requires thorough modelling which takes into account spatial kinematics of the turbulent disc and changes in the maser pumping conditions. This is beyond the scope of the present paper.

One possibility to tell the difference between the two hypotheses comes from the existence of the spectral features with velocities greatly different from the systemic velocity of the object: the maser velocities spread from $-93 \mathrm{~km} \mathrm{~s}^{-1}$ to $-17 \mathrm{~km} \mathrm{~s}^{-1}$ (Cohen 1979) while the systemic velocity is about $-48 \mathrm{~km} \mathrm{~s}^{-1}$. Recent sensitive observations of the water masers in W3(H2O) displayed features for which the difference in velocities with the bulk of the TW material reaches $70-90 \mathrm{~km} \mathrm{~s}^{-1}$ (Sobolev et al., private communication). The high velocity maser features can appear in the clumps which are torn off the surface close to the central parts of the disk. In this case maser spots corresponding to blue-shifted and red-shifted features are likely to have similar distribution in the field of view. Proper motions of the maser features under the disk hypothesis are determined by the proper motion of the TW object itself, the disk rotation and the turbulent motions at the disk surface. The procedure of estimating the TW object proper motion should be the same under both hypotheses and it is reasonable to assume that it is about $20 \mathrm{~km} \mathrm{~s}^{-1}$ as was estimated by Alcolea et al. (1993). The sense of the disk rotation can be guessed from the velocity gradient of the highly excited molecules in closest vicinity of the TW object observed by Wyrowski et al. (1997): it should be eastward for the blue-shifted and westward for the red-shifted features. The value of the rotation velocity for the material responsible for the high velocity features is uncertain. However, in the central vicinity of the disk this quantity can be considerably greater than both the proper motion of the TW object and the spread of the turbulent velocities. In this case the eastward direction of the blue-shifted maser spots and the westward direction of the red-shifted ones should be pronounced. The proper motion pattern in the case of the jet is discussed in the next subsection and is greatly different.

\subsection{Kinematics of water masers: Jet}

Alcolea et al. (1993) considered water maser kinematics under the hypothesis of bipolar outflow and found no major contradictions. We further consider the situation with high velocity maser features in the case of a jet. As was mentioned in the previous subsection, the possibility to discriminate between the two hypothesis can be provided by the interferometric measurements of positions and proper motions of the high velocity water maser features.

The results of Alcolea et al. (1993) show agreement with a considerable increase of the water maser velocities toward the center. In this case the high velocity features are expected to be situated close to the center. Observations of Wilner et al. (1999) show that the synchrotron feature wiggles. Anyhow, the observed jet convolution is not high and the maser kinematics shows that the central parts of the jet are most likely moving close to the tangential direction. In this case the proper motion of each spot producing high velocity maser feature should be much greater than the difference between the radial velocity of the feature and that of the TW object. These differences for the high velocity features considerably exceed the TW proper motion (about $20 \mathrm{~km} \mathrm{~s}^{-1}$ according to Alcolea et al. 1993). So in contrast to the case of the disk the proper motions of blue-shifted spots which trace the jet should be always great and should have different directions: westward for the spots to the west of the center and eastward for the spots to the east of the center. Further, in contrast to the case of the disk, the proper motion pattern for the red-shifted spots which trace the jet should be the same as that for the blue-shifted ones.

\subsection{Wiggling: Disk}

Wilner et al. (1999) have found that the synchrotron structure is not precisely straight, but rather wiggles at the WE ends with a remarkable point-like symmetry around the center. In the disk model a wiggling synchrotron structure may reflect a warped structure of the disk itself, which can be produced either by gravitational perturbation from companion stars or sufficiently massive gas clumps, or by a large-scale hydrodynamical instability driven by radiation from the central star (Pringle 1996; Armitage \& Pringle 1997).

Wyrowski et al. (1999) estimate the star embedded in the dust spot $\mathrm{C}$ to be a B0 star, and a total mass of dust emitting gas of $\sim 15 M_{\odot}$. The corresponding gravitational perturbation on the disk from both the star and the gas in the B and C clumps can be evaluated by comparing the vertical components of the gravitational force from the central star $\sim M H / r^{3}$, where $H$ is the vertical scale height of the disk, and from a companion star and surrounding gas $\sim\left(M_{\mathrm{c}}+M_{\mathrm{BC}}\right) a / r^{3}$, where $a$ is the distance between the disk plane and the center of mass of clumps $\mathrm{B}$ and $\mathrm{C}$ and an embedded star. The geometry of the TW source and of dust spots A, B and C suggests that $a \sim H$, which leads to the conclusion that the perturbation from dust clumps $\mathrm{B}, \mathrm{C}$ and the star is sufficient to warp the disk if $\left(M_{\mathrm{c}}+M_{\mathrm{BC}}\right) \sim M$.

A complementary source for keeping the disk warped may be connected with the interaction of the light from the central star with the disk: when the intrinsic luminosity of a central star settling onto the main sequence overwhelms the total disk luminosity, it can initiate a large scale instability and bend the disk (Pringle 1996; Armitage \& Pringle 1997). The threshold luminosity for the instability to grow is $\sim 10 L_{\odot}$, and is much less than the total luminosity of the stars embedded in the TW structure, $L_{\mathrm{TW}} \sim 3 \times 10^{4} L_{\odot}$ (Wyrowski et al. 1999). The warp 
excited initially at the inner disk diffuses outside and can last tens of Myrs. At later times, $t=15 \mathrm{Myr}$, the tilt angle between the inner $(r \sim 10 \mathrm{AU})$ and outer $(r \sim 100 \mathrm{AU})$ disk can reach $\sim 10^{\circ}$ (Armitage \& Pringle 1997). Armitage \& Pringle argue that this mechanism is most likely responsible for warping of the inner disk in $\beta$ Pictoris (Burrows et al. 1995). As shown by Quillen (2001) a strong stellar wind is also expected to produce disk warping.

A change of the wiggle morphology in the disk model can appear either due to relative motions of the central star (traced presumably by the A spot) and its companions (traced by B and $\mathrm{C}$ spots) if warping is produced by gravitational perturbation from these sources, or by proper rotation of the disk. In both cases the characteristic times are much longer than in the jet model: $t \sim \sqrt{G M / r^{3}} \sim 5 \times 10^{3} \mathrm{yr}$ for $M \sim 10 M_{\odot}$, and $r \sim 0.01 \mathrm{pc}$. This is consistent with observed lack of morphology variations over 510 days (Wilner et al. 1999).

\subsection{Wiggling: Jet}

Wilner et al. (1999) argue that wiggling of the synchrotron source in the TW may be the result of precession of the jet axis. In principle, precession is typical for jets in the vicinity of binary stellar objects and in AGNs. In the TW object precession can be maintained also by the gravitational influence from a companion star associated with the $\mathrm{B}$ and $\mathrm{C}$ dust spots. Large scale wiggling can be connected with a long-periodic precession with characteristic time $t \sim L / u$, where $L$ is the length of the jet, and $u$ is the speed of flow within it. The synchrotron structure in the TW object has an extent $2 L \sim 0.01 \mathrm{pc}$ (Reid et al. 1995), and the velocities of the water masers provide a lower estimate of the flow velocity: $u \sim 20 \mathrm{~km} \mathrm{~s}^{-1}$ at $1^{\prime \prime}$ and increasing to the center (Alcolea et al. 1993), $u \lesssim$ $40 \mathrm{~km} \mathrm{~s}^{-1}$ (Cohen 1979). Recent sensitive observations of the water masers in $\mathrm{W} 3(\mathrm{H} 2 \mathrm{O})$ displayed features for which the difference in velocities with the bulk of the TW material reaches $70-90 \mathrm{~km} \mathrm{~s}^{-1}$ (Sobolev et al., private communication). Thus, the upper limit for long-periodic variations of the wiggle morphology is $250 \mathrm{yr}$, while the lower limit can be not less than five years for the jet flow slower than $1000 \mathrm{~km} \mathrm{~s}^{-1}$ expected for the conditions in the vicinity of a young OB star. These estimates do not contradict the data of Wilner et al. (1999) on the absence of noticeable variability within 510 days.

\subsection{Polarization: Disk}

The jet and the accretion disk surface are expected to show different polarization properties. In the first case the emission is expected to be linearly polarized because it arises in the regions with an apparently stronger and rather regular magnetic field of the jet. In principle, the interface between the jet and the ambient medium is subject to the Kelvin-Helmholtz instability, which forces the interface to settle into a regime with highly developed turbulent motions causing depolarization. Indeed, numerical simulations show that super-Alfvénic jets undergo $\mathrm{KH}$ instabilities; however, only the relatively narrow region close to the jet walls becomes involved in a strongly nonlinear turbulent regime, while the body of the jet remains more regular since the characteristic growth rate of the instability there is an order of magnitude smaller than at the interface. As a result, the modelled synchrotron emission reveals a high degree of polarization (Hardee \& Rosen 1999).

Wilner et al. (1999) mention that morphologically the synchrotron structure in the TW source is similar to that observed in AGNs, where polarization in the jet component can reach $10 \%$. In some cases the degree of polarization in extragalactic jets can be as high as $50 \%$ - an upper limit for optically thin synchrotron emission (Perlman et al. 1999), which is consistent with the predictions of Hardee \& Rosen (1999).

In the case of synchrotron radiation produced in the boundary layer of the disk, where the acceleration of non-thermal electrons is intimately connected with a highly developed turbulence, the emission seems to be depolarized. Wilner et al. (1999) reported that the synchrotron source in the TW does not show polarization higher than 0.05 of the total intensity of the core of the synchrotron structure, and from this point of view it is consistent with the disk model.

\subsection{Polarization: Jet}

However, Wilner et al. bring forward several arguments explaining the nondetection of polarization from the TW synchrotron source. The fact that the core component does not show significant polarization might reflect the similarity between this source and the jets in ANGs where the core component emission shows typically less than a few percent of the total emission. Note, that in the case of the TW source it can be connected with a short periodic (a few days) variations of the core component produced by a strong influence of the magnetic field of the magnetosphere of the central star on the foot region of the jet, and highly developed random motions caused by this (see, e.g. Lovelace et al. 1995; Goodson et al. 1999). The jet component, where AGN jets normally show polarization, in the case of the TW source is too weak for the confident detection of polarization.

On the other hand, internal Faraday depolarization produced by thermal electrons with $n_{\mathrm{e}} \gtrsim 10^{3} \mathrm{~cm}^{-3}$ would be sufficient to explain the low level of polarization. The upper limit derived by Wilner et al. for $n_{\mathrm{e}}$ from the lack of detected thermal emission in the cm-wavelength range and from the size of the TW synchrotron structure is more than two orders of magnitude larger than this value. Under these circumstances a more precise determination of the thermal electron density would be of critical importance.

\section{Conclusions}

1. We have shown that synchrotron emission observed from the TW object can, in principle, be connected with the accretion disk around a massive star. Interaction of the wind and the strong shocks developed in the wind flow with the disk surface can generate multiple shocks in the boundary layer on the disk surface, which in turn can efficiently produce relativistic electrons. If only as little as than $0.5 \%$ of the wind kinetic energy is transformed into 
relativistic electrons, the total synchrotron luminosity can be comparable to that observed around the TW object in the $\mathrm{W} 3(\mathrm{OH})$ region.

2. The observed dependence of the angular size of the source on frequency can be explained as due to radial variation of the upper cutoff frequency of the synchrotron spectrum, which can be connected in particular with the radial dependence of the magnetic field in the disk.

3. We have shown that existing data on the spatial distribution of dust emission, kinematics of molecular material as well as morphology and polarization of the synchrotron emission around the TW object can be explained both with the disk and the jet hypothesis. Further observational efforts in the directions shown (e.g., measurement of proper motions of the water maser spots corresponding to the high velocity features) can distinguish between the two hypotheses.

In this paper we did considered synchrotron emission only around the TW object. Recently there have been reports of other sources of combined radiocontinuum and water maser emission associated with massive young stellar objects which probably contain disks (see Menten \& van der Tak 2004, and references therein). The bulk of the radiocontinuum emission around these sources looks different from synchrotron emission as can be inferred from their spectral indices. Thus, the suggested phenomenon can be widespread in the massive objects but difficult to observe because of confusion with other types of emission.

In our model the synchrotron luminosity of the circumstellar disk is proportional to the mass loss rate $\dot{M}$, the wind velocity $v$, and the disk radius $R$; it depends on the magnetic field slightly more strongly, as $B^{1.6}$. One can thus think that only the disks around massive stars with high enough $\dot{M}$ and $v$ can be sufficiently bright in synchrotron radiation. Note, however, that among the essential factors which can determine synchrotron luminosity are also the separation between the shock waves $l_{\mathrm{s}}$, and the spectrum of the turbulent magnetic field $\sim k^{-\xi}$; additional study is needed to draw firm conclusions on whether disks around less massive stars, such as for instance $\mathrm{T}$ Tauri, can shine in synchrotron radiation.

Acknowledgements. We thank the referee M. J. Reid and K. Menten for valuable critical comments. Stimulating discussions with I. I. Zinchenko are greatly acknowledged. Part of this work was supported by INTAS project 99-1667, RFBR projects 02-02-17642 and 03-02-16433, and grant of Ministry of Education of Russian Federation. Y.S. acknowledges financial support from Deutsche Forschungsgemeinschaft, DFG (project SFB N 591, TP A6).

\section{References}

Alcolea, J., Menten, K. M., Moran, J. M., \& Reid, M. J. 1993, in Astrophysical masers, Proceedings of the Conference, Arlington, VA, Mar. 9-11, 1992, 225

Armitage, P. J., \& Pringle, J. E. 1997, ApJ, 488, L47

Begelman, M. C., Blandford, R. D., \& Rees, M. J. 1984, Rev. Mod. Phys., 56, 255

Burrows, C. J., Krist, J. E., Stapelfeldt, K. R., et al. 1995, BAAS, 187, 32.05

Bykov, A. M., \& Fleishman, G. D. 1992, MNRAS, 256, 269

Carlberg, R. G. 1980, ApJ, 241, 1131

Cohen, N. L. 1979, Astrophys. Lett., 20, 81

Fiebig, D., Duschl, W. J., Menten, K. M., \& Tscharnuter, W. M. 1996 , A\&A, 310, 199

Fiebig, D., \& Güsten, R. 1989, A\&A, 214, 333

Ginzburg, V. L. 1975, Theoretical Physics and Astrophysics (Moscow: Nauka)

Goodson, A. P., Böhm, K.-H., \& Winglee, R. M. 1999, ApJ, 524, 142

Hardee, P. E., \& Rosen, A. 1999, ApJ, 524, 650

Head, M. R., \& Bandyopadhyay, P. 1981, J. Fluid Mech., 107, 297

Liljestroem, T., \& Gwinn, C. R. 2000, ApJ, 534, 781

Lovelace, R. V. E., Romanova, M. M., \& Bisnovatyi-Kogan, G. S. 1995, MNRAS, 275, 244

Lucy, L. B. 1982, ApJ, 255, 278

Menten, K. M., \& van der Tak, F. F. S. 2004, A\&A, 414, 289

Nelson, A. F., Benz, W., \& Ruzmaikina, T. V. 2000, ApJ, 529, 357

Owocki, S. P., \& Rybicki, G. B. 1984, ApJ, 284, 337

Perlman, E. S., Biretta, J. A., Zhou, F., Spark, W. B., \& Macchetto, F. D. 1999, AJ, 117, 2185

Pringle, J. E. 1981, ARA\&A, 19, 137

Pringle, J. E. 1996, MNRAS, 281, 357

Quillen, A. C. 2001, ApJ, 563, 313

Reid, M. J., Argon, A. L., Masson, C. R., Menten, K. M., \& Moran, J. M. 1995, ApJ, 443, 238

Roshko, A. 1976, AIAA J., 14, 1349

Sobolev, A. M., Sutton, E. C., Cragg, D. M., et al. 2001, Astron. Astrophys. Transact., 20, 229

Schwartz, R. D., \& Greene, T. P. 1999, AJ, 117, 456

Toptygin, I. N. 1985, Cosmic Rays in Interplanetary Magnetic Fields (Amsterdam: Reidel)

Torrelles, J. M., Patel, N. A., Gomez, J. F., \& Anglada, G. 2002, Rev. Mex. Astron. Astrofis. (Serie de Conferencias), 13, 108

Turner, J. L., \& Welch, W. J. 1984, ApJ, 287, L81

White, R. L. 1985, ApJ, 289, 698

Wilner, D. J., Reid, M. J., \& Menten, K. M. 1999, ApJ, 513, 775

Wyrowski, F., Hofner, P., Schilke, P., et al. 1997, A\&A, 320, L17

Wyrowski, F., Schilke, P., Walmsley, C. M., \& Menten, K. M. 1999, ApJ, 514, L43

Zank, G. P., Axford, W. I., \& McKenzie, J. F. 1990, A\&A, 233, 275 